This item was submitted to Loughborough's Research Repository by the author.

Items in Figshare are protected by copyright, with all rights reserved, unless otherwise indicated.

\title{
Universal formula for the Hilbert series of minimal nilpotent orbits
}

PLEASE CITE THE PUBLISHED VERSION

https://doi.org/10.1090/proc/13819

PUBLISHER

(c) American Mathematical Society

VERSION

AM (Accepted Manuscript)

PUBLISHER STATEMENT

This work is made available according to the conditions of the Creative Commons Attribution-NonCommercialNoDerivatives 4.0 International (CC BY-NC-ND 4.0) licence. Full details of this licence are available at: https://creativecommons.org/licenses/by-nc-nd/4.0/

\section{LICENCE}

CC BY-NC-ND 4.0

\section{REPOSITORY RECORD}

Matsuo, A., and Alexander Veselov. 2019. "Universal Formula for the Hilbert Series of Minimal Nilpotent Orbits". figshare. https://hdl.handle.net/2134/25427. 


\title{
UNIVERSAL FORMULA FOR HILBERT SERIES OF MINIMAL NILPOTENT ORBITS
}

\author{
A. MATSUO AND A.P. VESELOV
}

\begin{abstract}
We show that the Hilbert series of the projective variety $X=P\left(O_{\min }\right)$, corresponding to the minimal nilpotent orbit $\mathcal{O}_{\text {min }}$, is universal in the sense of Vogel: it is written uniformly for all simple Lie algebras in terms of Vogel's parameters $\alpha, \beta, \gamma$ and represents a special case of the generalized hypergeometric function ${ }_{4} F_{3}$. A universal formula for the degree of $X$ is then deduced.
\end{abstract}

\section{INTRODUCTION}

Let $\mathfrak{g}$ be a complex simple Lie algebra and $\mathcal{O}_{\text {min }}$ be the minimal (in the sense of dimension) non-zero nilpotent orbit in $\mathfrak{g} \approx \mathfrak{g}^{*}$. It is well-known that such orbit is unique, see [5]).

Its projective version $X=P\left(\mathcal{O}_{\text {min }}\right) \subset P(\mathfrak{g})$ is a smooth projective variety, sometimes called adjoint variety (see e.g. [10]). It is the only compact orbit of the corresponding complex group $G$ acting on $P(\mathfrak{g})$.

These varieties have some remarkable geometric properties. In particular, they can be characterised as compact, simply connected, contact homogeneous varieties [3], or, under certain assumptions, as Fano contact manifolds [2]. Their quantum versions related to Joseph ideals [9] were studied in [12].

In this note we analyse these varieties from the point of view of the universal simple Lie algebra of Vogel [14]. This object is still to be defined, but Vogel's parametrisation of simple Lie algebras has already proved to be very useful for representation theory (see e.g. $[11,13]$ ).

Recall that the universal Vogel's parameters $\alpha, \beta, \gamma$ can be defined as follows [14]. Take symmetric part of the tensor square of the adjoint representation of $\mathfrak{g}$ and decompose it into irreducible representations. Then it turns out that, apart from a trivial representation corresponding to invariant symmetric bilinear form, there will be three of them (two for the exceptional Lie algebras).

Choose an invariant bilinear form on $\mathfrak{g}$ and consider the eigenvalues of the corresponding Casimir element on these representations. In Vogel's parametrisation they are $4 t-2 \alpha, 4 t-2 \beta, 4 t-2 \gamma$, where $t=\alpha+\beta+\gamma$, which defines the parameters uniquely up to a common multiple (because of the freedom in the choice of the invariant form). If we choose the normalisation in which the negative parameter $\alpha=-2$, then $t=h^{\vee}$ is the dual Coxeter number and the corresponding parameters are given in Table 1. 


\begin{tabular}{|c|c|c|c|c|c|}
\hline Type & Lie algebra & $\alpha$ & $\beta$ & $\gamma$ & $t=h^{\vee}$ \\
\hline$A_{n}$ & $\mathfrak{s l}_{n+1}$ & -2 & 2 & $n+1$ & $n+1$ \\
$B_{n}$ & $\mathfrak{s o}_{2 n+1}$ & -2 & 4 & $2 n-3$ & $2 n-1$ \\
$C_{n}$ & $\mathfrak{s p}_{2 n}$ & -2 & 1 & $n+2$ & $n+1$ \\
$D_{n}$ & $\mathfrak{s o}_{2 n}$ & -2 & 4 & $2 n-4$ & $2 n-2$ \\
$E_{6}$ & $\mathfrak{e}_{6}$ & -2 & 6 & 8 & 12 \\
$E_{7}$ & $\mathfrak{e}_{7}$ & -2 & 8 & 12 & 18 \\
$E_{8}$ & $\mathfrak{e}_{8}$ & -2 & 12 & 20 & 30 \\
$F_{4}$ & $\mathfrak{f}_{4}$ & -2 & 5 & 6 & 9 \\
$G_{2}$ & $\mathfrak{g}_{2}$ & -2 & $10 / 3$ & $8 / 3$ & 4 \\
\hline
\end{tabular}

TABLE 1. Vogel's parameters for simple Lie algebras

Now a formula for numerical characteristics of a simple Lie algebra is called universal if it is written in terms of Vogel's parameters.

In this note, we will give some universal formulae related to the minimal nilpotent orbit $\mathcal{O}_{\min } \subset \mathfrak{g}$ by means of the Hilbert series of its projectivisation $X=P\left(\mathcal{O}_{\text {min }}\right) \subset P(\mathfrak{g})$.

Recall that for a projective variety $X \subset \mathbb{P}^{n}$ the Hilbert series $H_{X}(z)$ is defined as the generating function

$$
H_{X}(z)=\sum_{k=0}^{\infty} \operatorname{dim}\left(S(X)_{k}\right) z^{k},
$$

where $S(X)=\mathbb{C}\left[x_{0}, \ldots, x_{n}\right] / I(X)$ is the homogeneous coordinate ring of $X$ and $S(X)_{k}$ is the component of degree $k$. The $\operatorname{dimension} \operatorname{dim}\left(S(X)_{k}\right)$ for large $k$ is written as

$$
\operatorname{dim}\left(S(X)_{k}\right)=h_{X}(k)
$$

by a polynomial $h_{X}(x)$ called the Hilbert polynomial, see [8].

Now, return to the case when $X=P\left(\mathcal{O}_{\min }\right)$ and introduce the parameters given by

$$
\begin{gathered}
a_{1}=2 b_{1}+2 b_{2}-3, a_{2}=b_{1}+2 b_{2}-2, a_{3}=2 b_{1}+b_{2}-2, \\
b_{1}=-\frac{\beta}{\alpha}, b_{2}=-\frac{\gamma}{\alpha},
\end{gathered}
$$

where $\alpha, \beta, \gamma$ are Vogel's parameters given in Table 1. Recall that the Pochhammer symbol $(a)_{n}$ is defined as

$$
(a)_{n}=a(a+1) \ldots(a+n-1), \quad n=1,2, \ldots
$$

with $(a)_{0}=1$. Then our main observation is the following universal formula describing the Hilbert series of $X$. 
Theorem 1.1. The Hilbert series of the projectivisation $X=P\left(\mathcal{O}_{\min }\right)$ of the minimal nilpotent orbit $\mathcal{O}_{\min }$ has the following universal form

$$
H_{X}(z)=\sum_{k=0}^{\infty}\left(1+\frac{2 k}{a_{1}}\right) \frac{\left(a_{1}\right)_{k}\left(a_{2}\right)_{k}\left(a_{3}\right)_{k}}{\left(b_{1}\right)_{k}\left(b_{2}\right)_{k} k !} z^{k},
$$

with the parameters $a_{1}, a_{2}, a_{3}, b_{1}, b_{2}$ given in terms of Vogel's parameters by (2) and (3).

In the rest of the note we will derive the formula (5) and describe its consequences. In particular, we will extract universal formulae for the dimension and the degree of the projective variety $X$. The explicit values of the parameters $a_{1}, a_{2}, a_{3}, b_{1}, b_{2}$ as well as $\operatorname{dim} X$ and $\operatorname{deg} X$ are listed in Table 2.

\begin{tabular}{|c|c|c|c|c|c||c|c|}
\hline Type & $a_{1}$ & $a_{2}$ & $a_{3}$ & $b_{1}$ & $b_{2}$ & $\operatorname{dim} X$ & $\operatorname{deg} X$ \\
\hline$A_{n}$ & $n$ & $n$ & $\frac{n+1}{2}$ & 1 & $\frac{n+1}{2}$ & $2 n-1$ & $\left(\begin{array}{c}2 n \\
n\end{array}\right)$ \\
$B_{n}$ & $2 n-2$ & $2 n-3$ & $n+\frac{1}{2}$ & 2 & $n-\frac{3}{2}$ & $4 n-5$ & $\frac{4}{2 n-1}\left(\begin{array}{l}4 n-4 \\
2 n-2\end{array}\right)$ \\
$C_{n}$ & $n$ & $n+\frac{1}{2}$ & $\frac{n}{2}$ & $\frac{1}{2}$ & $\frac{n}{2}+1$ & $2 n-1$ & $2^{2 n-1}$ \\
$D_{n}$ & $2 n-3$ & $2 n-4$ & $n$ & 2 & $n-2$ & $4 n-7$ & $\frac{4}{2 n-2}\left(\begin{array}{l}4 n-6 \\
2 n-3\end{array}\right)$ \\
$E_{6}$ & 11 & 9 & 8 & 3 & 4 & 21 & 151164 \\
$E_{7}$ & 17 & 14 & 12 & 4 & 6 & 33 & 141430680 \\
$E_{8}$ & 29 & 24 & 20 & 6 & 10 & 57 & 126937516885200 \\
$F_{4}$ & 8 & $\frac{13}{2}$ & 6 & $\frac{5}{2}$ & 3 & 15 & 4992 \\
$G_{2}$ & 3 & $\frac{7}{3}$ & $\frac{8}{3}$ & $\frac{5}{3}$ & $\frac{4}{3}$ & 5 & 18 \\
\hline
\end{tabular}

TABle 2. Parameters, dimension and degree of $X=P\left(\mathcal{O}_{\text {min }}\right)$.

\section{UNIVERSAl FORMUla FOR HILBERT SERIES}

The derivation of the universal formula (5) is in fact simply a combination of two important results.

The first one goes back to Borel and Hirzebruch and sometime is attributed to Kostant, see [6,7]. It claims that as a $\mathfrak{g}$-module $S(X)$ for $X=P\left(\mathcal{O}_{\text {min }}\right)$ has a form

$$
S(X)=\bigoplus_{k=0}^{\infty} V(k \theta)
$$

where $\theta$ is the maximal root of $\mathfrak{g}$ and $V(\lambda)$ is the irreducible representation with the highest weight $\lambda$ (see [6]). 
The second one is the universal formula for the dimension of $V(k \theta)$ found by Landsberg and Manivel [11]:

$$
\operatorname{dim} V(k \theta)=\frac{t-\left(k-\frac{1}{2}\right) \alpha}{t+\frac{\alpha}{2}} \frac{\left(\begin{array}{c}
-\frac{2 t}{\alpha}-2+k \\
k
\end{array}\right)\left(\begin{array}{c}
\frac{\beta-2 t}{\alpha}-1+k \\
k
\end{array}\right)\left(\begin{array}{c}
\frac{\gamma-2 t}{\alpha}-1+k \\
k
\end{array}\right)}{\left(\begin{array}{c}
-\frac{\beta}{\alpha}-1+k \\
k
\end{array}\right)\left(\begin{array}{c}
-\frac{\gamma}{\alpha}-1+k \\
k
\end{array}\right)},
$$

where $\left(\begin{array}{l}a \\ k\end{array}\right)$ is a binomial coefficient defined for any $a$ and integer $k \geq 0$ as

$$
\left(\begin{array}{l}
a \\
k
\end{array}\right)=\frac{(a-k+1) \ldots(a-1) a}{k !} .
$$

Rewriting (7) in terms of Pochhammer symbols (4) and combining it with (6), we have

$$
\operatorname{dim} S(X)_{k}=\left(1+\frac{2 k}{a_{1}}\right) \frac{\left(a_{1}\right)_{k}\left(a_{2}\right)_{k}\left(a_{3}\right)_{k}}{\left(b_{1}\right)_{k}\left(b_{2}\right)_{k} k !}
$$

where $a_{1}, a_{2}, a_{3}, b_{1}, b_{2}$ are given by (2) and (3). This gives us formula (5).

Let us now turn to the Hilbert polynomial $h_{X}(x)$ which satisfies

$$
h_{X}(k)=\operatorname{dim} S(X)_{k}
$$

if $k$ is sufficiently large. To obtain a universal formula for $h_{X}(x)$, apply the formula

$$
(a)_{k}=\frac{\Gamma(a+k)}{\Gamma(a)}
$$

where $\Gamma(x)$ is the classical Gamma-function [16]. Then the right-hand side of (8) becomes

$$
\frac{\Gamma\left(b_{1}\right) \Gamma\left(b_{2}\right)}{\Gamma\left(a_{1}\right) \Gamma\left(a_{2}\right) \Gamma\left(a_{3}\right)}\left(1+\frac{2 k}{a_{1}}\right) \frac{\Gamma\left(a_{1}+k\right) \Gamma\left(a_{2}+k\right) \Gamma\left(a_{3}+k\right)}{\Gamma\left(b_{1}+k\right) \Gamma\left(b_{2}+k\right) \Gamma(1+k)} .
$$

Since this expression for the parameters corresponding to simple Lie algebras turns out to be a polynomial in $k$, we have

Corollary 2.1. The Hilbert polynomial of $X=P\left(\mathcal{O}_{\text {min }}\right)$ has the following universal form

$$
h_{X}(x)=\frac{\Gamma\left(b_{1}\right) \Gamma\left(b_{2}\right)}{\Gamma\left(a_{1}\right) \Gamma\left(a_{2}\right) \Gamma\left(a_{3}\right)}\left(1+\frac{2 x}{a_{1}}\right) \frac{\Gamma\left(a_{1}+x\right) \Gamma\left(a_{2}+x\right) \Gamma\left(a_{3}+x\right)}{\Gamma\left(b_{1}+x\right) \Gamma\left(b_{2}+x\right) \Gamma(1+x)},
$$

with $h_{X}(k)=\operatorname{dim}\left(S(X)_{k}\right)$ for all $k \geq 0$.

The fact that the last formula works for all $k \geq 0$ follows from [7].

\section{Geometric consequences}

Recall that Hilbert polynomial of $X$ determines both the dimension and the degree of $X$ as

$$
h_{X}(x)=\operatorname{deg} X \frac{x^{d}}{d !}+\ldots,
$$

where $d=\operatorname{dim} X$ (see e.g. [7]). Applying the limit

$$
\lim _{x \rightarrow+\infty} \frac{\Gamma(a+x)}{\Gamma(x)} x^{-a}=1
$$


to (9), we have the asymptotic expansion

$$
h_{X}(x)=A x^{a_{1}+a_{2}+a_{3}-b_{1}-b_{2}}+\ldots,
$$

where the leading coefficient $A$ is given by

$$
A=\frac{2 \Gamma\left(b_{1}\right) \Gamma\left(b_{2}\right)}{a_{1} \Gamma\left(a_{1}\right) \Gamma\left(a_{2}\right) \Gamma\left(a_{3}\right)} .
$$

Therefore, we have $\operatorname{dim} X=d=a_{1}+a_{2}+a_{3}-b_{1}-b_{2}$ and $\operatorname{deg} X=A \cdot d$ ! by (10). After a simple algebra, we obtain

Corollary 3.1. The dimension and the degree of $X=P\left(\mathcal{O}_{\text {min }}\right)$ are given by

$$
\begin{gathered}
\operatorname{dim} X=2 a_{1}-1, \\
\operatorname{deg}(X)=\frac{2 \Gamma\left(2 a_{1}\right) \Gamma\left(b_{1}\right) \Gamma\left(b_{2}\right)}{\Gamma\left(a_{1}+1\right) \Gamma\left(a_{2}\right) \Gamma\left(a_{3}\right)}
\end{gathered}
$$

respectively. by

Since $2 a_{1}-1=-\frac{4 t}{\alpha}-3=2 h^{\vee}-3$, the dimension is more explicitly given

$$
\operatorname{dim} X=2 h^{\vee}-3
$$

where $h^{\vee}$ is the dual Coxeter number of $\mathfrak{g}$. This recovers the formula due to Wang [15]:

$$
\operatorname{dim} \mathcal{O}_{\min }=2 h^{\vee}-2 .
$$

Let us compare this with the results of Gross and Wallach [7], who derived from the Weyl dimension formula that Hilbert polynomial $h_{X}(q)$ can be given as a product over positive roots of $\mathfrak{g}$ :

$$
h_{X}(q)=\prod_{\alpha \in R_{+}}\left(1+\frac{\left(\theta, \alpha^{\vee}\right)}{\left(\rho, \alpha^{\vee}\right)} q\right),
$$

where $\rho$ is the half-sum of the positive roots. The Hilbert series of $X$ has the form

$$
H_{X}(z)=h_{X}\left(z \frac{d}{d z}\right) \frac{1}{1-z} .
$$

As a result they arrived at the following formula for the degree of $X$ :

$$
\operatorname{deg}(X)=d ! \prod_{\alpha} \frac{\left(\theta, \alpha^{\vee}\right)}{\left(\rho, \alpha^{\vee}\right)},
$$

where the product is taken over positive roots such that $\left(\theta, \alpha^{\vee}\right) \neq 0$. It would be interesting to deduce from here our formula (13), which is a universal form of the right hand side of (16).

Let us consider as an example the $A_{n}$-case, when $X=P\left(\mathcal{O}_{\text {min }}\right)$ is the hyperplane section of the Segre variety $[7,8]$. The formula (13) gives in this case

$$
\operatorname{deg} X=\frac{2 \Gamma(2 n) \Gamma(1) \Gamma\left(\frac{n+1}{2}\right)}{\Gamma(n+1) \Gamma(n) \Gamma\left(\frac{n+1}{2}\right)}=\frac{2 \cdot(2 n-1) !}{n !(n-1) !}=\left(\begin{array}{c}
2 n \\
n
\end{array}\right),
$$

which agrees with well-known result (see [8]). 
The degrees of other types are presented in Table 2 . The result in $E_{8}$-case agrees with the one given in [7]. It is interesting to note that in type $B$ and $D$ the degrees are the corresponding Catalan numbers times 4 .

\section{Hilbert SERIES AS HYPERGEOMETRIC FUnCTION}

Let us show that the Hilbert series of $X=P\left(\mathcal{O}_{\text {min }}\right)$ is in fact a particular case of the generalized hypergeometric function ${ }_{4} F_{3}$ (cf. [1]):

$$
{ }_{4} F_{3}\left(a_{1}, a_{2}, a_{3}, a_{4} ; b_{1}, b_{2}, b_{3} ; z\right)=\sum_{n=0}^{\infty} \frac{\left(a_{1}\right)_{n}\left(a_{2}\right)_{n}\left(a_{3}\right)_{n}\left(a_{4}\right)_{n}}{\left(b_{1}\right)_{n}\left(b_{2}\right)_{n}\left(b_{3}\right)_{n}} \frac{z^{n}}{n !} .
$$

To see this, let the parameters $a_{1}, a_{2}, a_{3}, b_{1}, b_{2}$ be given by (2), (3), and $a_{4}, b_{3}$ by

$$
a_{4}=b_{3}+1, b_{3}=-\frac{2 t+\alpha}{2 \alpha}=\frac{a_{1}}{2} .
$$

Then (8) is rewritten as

$$
\operatorname{dim} S(X)_{k}=\frac{\left(a_{1}\right)_{k}\left(a_{2}\right)_{k}\left(a_{3}\right)_{k}\left(a_{4}\right)_{k}}{\left(b_{1}\right)_{k}\left(b_{2}\right)_{k}\left(b_{3}\right)_{k} k !}
$$

and hence we obtain

Corollary 4.1. The Hilbert series of $X=P\left(\mathcal{O}_{\text {min }}\right)$ is written as

$$
H_{X}(z)={ }_{4} F_{3}\left(a_{1}, a_{2}, a_{3}, a_{4} ; b_{1}, b_{2}, b_{3} ; z\right),
$$

with the parameters $a_{1}, a_{2}, a_{3}, a_{4}, b_{1}, b_{2}, b_{3}$ given by (2), (3) and (18).

Note that the series can also be written in terms of ${ }_{3} F_{2}$ hypergeometric function as

$$
H_{X}(z)=\left(1+\frac{2}{a_{1}} z \frac{d}{d z}\right){ }_{3} F_{2}\left(a_{1}, a_{2}, a_{3} ; b_{1}, b_{2} ; z\right) .
$$

We can well use the formula (20) to derive the dimension formula for the variety $X$. Recall the differential equation for the hypergeometric function $F={ }_{4} F_{3}$ :

$$
\left[D \prod_{j=1}^{3}\left(z D+b_{j}-1\right)-\prod_{j=1}^{4}\left(z D+a_{j}\right)\right] F=0, \quad D=\frac{d}{d z} .
$$

The point $z=1$ is a regular singular point (in the sense of analytic theory of differential equations) with the exponents $0,1,2$ and

$$
s=b_{1}+b_{2}+b_{3}-a_{1}-a_{2}-a_{3}-a_{4}
$$

(see e.g. [4]). Since the Hilbert series $H_{X}(z), X=P\left(\mathcal{O}_{\min }\right)$, is a rational function with the pole at $z=1$ of order equal to the dimension of $\operatorname{dim} X+1$, we have $\operatorname{dim} X=-s-1=a_{1}+a_{2}+a_{3}+a_{4}-b_{1}-b_{2}-b_{3}-1=2 h^{\vee}-3$.

Finally, let us recall that the Hilbert series $H_{X}(z)$ can be written as

$$
H_{X}(z)=\frac{P_{X}(z)}{(1-z)^{d+1}}
$$


for some palindromic polynomial $P_{X}(z)$ with $P_{X}(1)=\operatorname{deg} X$ (see e.g. [7]). In our case, the problem of finding such a $P_{X}(z)$ is a particular case of the connection problem for the differential equation (22) from $z=0$ to $z=1$, but formally we can write it down universally as

$$
P_{X}(z)=(1-z)^{d+1} H_{X}(z)\left(\bmod (z-1)^{d+1}\right),
$$

where $H_{X}(z)$ is given by $(20)$.

\section{Concluding Remarks}

Our results imply also that the Hilbert series of the ideal $I=I(X)$ determining $X$

$$
H_{I}(z)=\sum_{k=0}^{\infty} \operatorname{dim} I_{k} z^{k}
$$

where $I_{k}$ is the component of $I$ of degree $k$, is also universal. Indeed we have

$$
H_{I}(z)=\frac{1}{(1-z)^{N}}-H_{X}(z),
$$

and $N=\operatorname{dim} \mathfrak{g}$ is the dimension of $\mathfrak{g}$, which can be given by Vogel's formula

$$
N=\frac{(\alpha-2 t)(\beta-2 t)(\gamma-2 t)}{\alpha \beta \gamma}=\frac{\left(a_{1}+2\right) a_{2} a_{3}}{b_{1} b_{2}} .
$$

Since

$$
\frac{1}{(1-z)^{N}}=\sum_{k=0}^{\infty}\left(\begin{array}{c}
-N \\
k
\end{array}\right)(-z)^{k}=\sum_{k=0}^{\infty} \frac{(N)_{k}}{k !} z^{k},
$$

this implies the following universal expression for $\operatorname{dim} I_{k}$ :

$$
\operatorname{dim} I_{k}=\frac{(N)_{k}}{k !}-\left(1+\frac{2 k}{a_{1}}\right) \frac{\left(a_{1}\right)_{k}\left(a_{2}\right)_{k}\left(a_{3}\right)_{k}}{\left(b_{1}\right)_{k}\left(b_{2}\right)_{k} k !} .
$$

Note that all our formulae are symmetric in $\beta$ and $\gamma$, but not in $\alpha$. A natural question is about the meaning of the corresponding Hilbert series when we permute $\alpha$ with $\beta$ or $\gamma$. From Landsberg and Manivel [11] we have an interpretation of the corresponding coefficients as the dimensions of certain virtual $\mathfrak{g}$-modules, which are zero for large $k$ (see the full list for all types in Section 6 of [11]).

According to (12),(13) the corresponding "virtual varieties" $Y$ and $Z$ must have degree 0 and negative dimensions:

$$
\operatorname{dim} Y=-\frac{4 t}{\beta}-3, \quad \operatorname{dim} Z=-\frac{4 t}{\gamma}-3 .
$$

In particular, for $A_{n}$ type

$$
\operatorname{dim} Y=-2 n-5, \operatorname{dim} Z=-7 .
$$

Is there any geometry behind this? 


\section{AcKnowledgements}

We are grateful to Alexey Bolsinov and Jenya Ferapontov for useful discussions.

The work of AM was partially supported by JSPS KAKENHI Grant Number JP26610004.

\section{REFERENCES}

[1] G.E. Andrews, R. Askey, R. Roy Special Functions. Cambridge Univ. Press, 2000.

[2] A. Beauville Fano contact manifolds and nilpotent orbits. Comment. Math. Helv. 73 (1998), 566-583.

[3] W.M. Boothby A note on homogeneous complex contact manifolds. Proc. AMS 13 (1962), 276-280.

[4] F. Beukers and G. Heckman Monodromy for the hypergeometric function ${ }_{n} F_{n-1}$. Invent. Math. 95 (1989), 325-354.

[5] D.H. Collingwood and W.M. McGovern Nilpotent orbits in semisimple Lie algebras. Van Nostrand Reinhold Math. Series, 1992.

[6] D. Garfinkle A new construction of the Joseph ideal. PhD thesis, MIT, 1982.

[7] B.H. Gross and N.R. Wallach On the Hilbert polynomials and Hilbert series of homogeneous projective varieties. Arithmetic geometry and automorphic forms, 253-263, Adv. Lect. Math. (ALM), 19, Int. Press, Somerville, MA, 2011.

[8] J. Harris Algebraic Geometry. A First Course. Springer, 1995.

[9] A. Joseph The minimal orbit in a simple Lie algebra and its associated maximal ideal. Ann. Sci. École Norm. Sup. (4), 9(1) (1976),1-29.

[10] H. Kaji, M. Ohno and O. Yasukura Adjoint varieties and their secant varieties. Indag. Mathem. N.S. 10 (1) (1999), 45-57.

[11] J.M. Landsberg, L. Manivel A universal dimension formula for complex simple Lie algebras. Adv. Math. 201 (2006), 379-407.

[12] T. Levasseur, S.P. Smith and J.T. Stafford The minimal nilpotent orbit, the Joseph ideal, and differential operators. J. Algebra 116 (1988), 480-501.

[13] R.L. Mkrtchyan, A.N. Sergeev, A.P. Veselov Casimir eigenvalues for universal Lie algebra. J. Math. Phys. 53(10) (2012),102106.

[14] P. Vogel The universal Lie algebra. Preprint (1999).

[15] W. Wang Dimension of a minimal nilpotent orbit. Proc. Amer. Math. Soc. 127(3) (1999), 935-936.

[16] E.T. Whittaker and G.N. Watson A Course of Modern Analysis. Cambridge University Press, 1963.

Graduate School of Mathematical Sciences, The University of Tokyo, Komaba, TOKYO, 153-8914, JAPAN

E-mail address: matsuo@ms.u-tokyo.ac.jp

Department of Mathematical Sciences, Loughborough University, LoughBorough LE11 3TU, UK and Moscow State University, Moscow 119899, Russia

E-mail address: A.P.Veselov@lboro.ac.uk 